\title{
Glottopol
}

Revue de sociolinguistique en ligne

$35 \mid 2021$

La langue à l'école, de l'institution à la classe : quelles conceptions, quelles normes, pour quels usages?

\section{Catherine Adam, 2020, Bilinguisme scolaire}

Familles, écoles, identités en Bretagne, éd. Peter Lang, coll. « Langue, multilinguisme et changement social », Berlin.

Marie-Jeanne Verny

\section{OpenEdition}

\section{Journals}

Édition électronique

URL : https://journals.openedition.org/glottopol/314

DOI : $10.4000 /$ glottopol.314

ISSN : $1769-7425$

Éditeur

Presses universitaires de Rouen et du Havre

Référence électronique

Marie-Jeanne Verny, "Catherine Adam, 2020, Bilinguisme scolaire », Glottopol [En ligne], 35 | 2021, mis en ligne le 01 janvier 2021, consulté le 02 octobre 2021. URL : http://journals.openedition.org/ glottopol/314; DOI : https://doi.org/10.4000/glottopol.314 


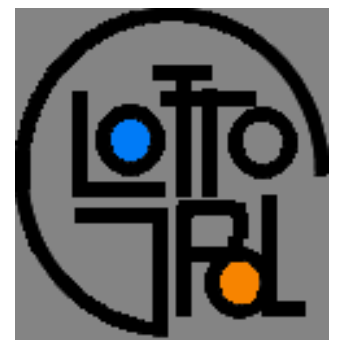

\section{GLOTTOPOL}

Revue de sociolinguistique en ligne $\mathrm{n}^{\circ} 35$ - janvier 2021

\section{La langue à l'école, de l'institution à la classe : quelles conceptions, quelles normes, pour quels usages?}

\section{SOMMAIRE}

Catherine Delarue-Breton et Élisabeth Bautier : Introduction

Catherine Delarue-Breton : Des conceptions scolaires de la langue en tension : l'exemple du programme de 2015 pour l'école primaire et le collège

Véronique Miguel-Addisu : "Sport de riche je l'ai changé en sport élitiste » : plurilinguisme et variation dans la langue de l'école du point de vue des élèves

Claire Colombel-Teuira, Véronique Fillol : Variation et approche polynomique : pour une conception plurielle de la langue à l'école calédonienne

Daphné Bloch : Pratiques langagières, situations pédagogiques et construction d'inégalités d'apprentissage à Madagascar

Samatar Abdallah Doualeh : Le français langue de l'école djiboutienne ou l'hégémonie de la norme exogène

Élise Vinel, Élisabeth Bautier : Des discours des élèves sur l'orthographe aux pratiques des enseignants, analyse d'entretiens métagraphiques

Thierry Pagnier, Belinda Lavieu-Gwozdz: Regards sur le discours scolaire: saisir des conceptions de la langue et de son enseignement

Jacques Crinon, Georges Ferone, Hélène Font : Les enseignants et l'orthographe, une enquête au cycle 3

Christel Troncy : Les enseignants, la norme scolaire et la pluralité langagière dans deux écoles immersives à programme français en Californie. Dynamiques des attitudes et des pratiques lors d'une recherche collaborative

Erwan Le Pipec: L'école, planche de salut du breton?

Argia Olçomendy : La langue basque dans les instructions officielles (1976-2019)

\section{Compte rendu de lecture}

Par Katrin Pfadenhauer: Ursula Reutner (Ed.), 2017, Manuel des francophonies, Berlin/Boston, de Gruyter, 745 p.

Par Robert Fournier: Shana Poplack, 2018, Borrowing: Loanwords in the speech community and in the grammar. New York: Oxford University Press ; xxi, $246 \mathrm{p}$.

Par Marie-Jeanne Verny: Catherine Adam, 2020, Bilinguisme scolaire. Familles, écoles, identités en Bretagne, éd. Peter Lang, coll. «Langue, multilinguisme et changement social », Berlin. 


\section{COMPTE RENDU}

Shana Poplack, 2018, Borrowing: Loanwords in the speech community and in the grammar. New York: Oxford University Press ; xxi, 246 p.

par Robert Fournier

\section{Carleton University, Ottawa}

Pour qui n'est pas au moins un peu familier avec les notions de sociolinguistique variationniste - communauté linguistique, (la distinction entre) code-switching (alternance codique), borrowing, loanword, nonce borrowing -, cet ouvrage paraitra au premier abord rébarbatif. Mais pour qui osera malgré tout en faire la lecture, l'aventure sera très enrichissante. Car, Shana Poplack est la grande spécialiste incontestée de tous ces sujets, pris séparément ou en combinaison. Elle est aussi une excellente pédagogue, et surtout une grande chercheure. Une difficulté supplémentaire, pour le lecteur francophone, est la confusion terminologique possible entrainée par la signification des termes borrowing (processus, et, mot emprunté de la langue donatrice (LD)), loanwoard (emprunt par la langue réceptrice (LR)), lone borrowing (emprunt isolé), nonce borrowing (emprunt à occurrence unique), que je donne ici à titre indicatif, mais qui en contexte ne devraient pas poser trop de problèmes malgré tout. À ce sujet, l'anglais n'est pas exempt de pareille confusion terminologique (Jaafar et al. 2019). Clairement, Poplack définit loanword comme étant le terme généralement réservé au produit du processus de l'emprunt (borrowing process), alors que borrowing "refers to items that have not (yet) achieved this status" (p. 7). Est-ce plus clair?

Mais Borrowing n'est pas un ouvrage pédagogique; il est l'aboutissement de plusieurs décennies de recherche de l'auteure sur le contact linguistique. Pieter Muysken a raison d'écrire dans l'avant-propos de l'ouvrage que « There can be no doubt that this is the most coherent, authoritative, and comprehensive study of the process of lexical borrowing so far undertaken, and it is hard to imagine another book superseding it in the years to come " (Foreword, xiii).

L'ouvrage s'appuie principalement sur un corpus irréprochable hautement stratifié recueilli dans la région bilingue d'Hull-Ottawa au début des années 1980, dont nous connaissons la composition depuis Poplack (1989), tous les détails étant fournis à l'annexe A (217-222) : 120 locuteurs de 4 sous-localités de ces deux villes voisines sur la frontière Québec-Ontario de la capitale fédérale ; 270 heures d'enregistrement de parole spontanée ; environ 2,5 millions de mots. La présente étude s'appuie aussi en partie sur deux autres corpus reliés historiquement et géographiquement au premier, et qui serviront aux analyses du chapitre 8, l'un, Récits du français québécois d'autrefois, un corpus recueilli par des folkloristes dans les années 1940 et 1950 auprès de locuteurs québécois âgés, nés entre 1846 et 1895, source précieuse de données du français du Québec rural du $19^{\mathrm{e}}$ siècle donc ; l'autre, le Corpus de français en contexte : 
milieux scolaire et social, un corpus recueilli entre 2005 et 2007 dans une école de la souslocalité du Mont Bleu, auprès de 166 adolescents francophones bilingues. Ces trois corpus réunis représentent en temps réel une période de 61 ans (1946-2007), et une étendue en temps apparent de presqu'un siècle et demi entre les dates de naissance des plus anciens et des plus jeunes locuteurs (1846-1994). Pour Poplack qui tient mordicus à appuyer empiriquement ses analyses, cela nous semble bien suffisant! Mais ce n'est pas tout, une large variété de minicorpus de paires de langues typologiquement distinctes (ukrainien-anglais, japonais-anglais, tamil-anglais, wolof-français, fongbe-français, etc.), et un "triplé » (vietnamien-françaisanglais) serviront à reproduire les découvertes faites à partir du corpus principal du français parlé à Hull-Ottawa, et ainsi valider les contraintes proposées sur la base de la paire françaisanglais typologiquement semblable. Les analyses proposées dans cet ouvrage s'appuieront donc sur la parole spontanée de 323 locuteurs bilingues français-anglais, plus 174 autres locuteurs bilingues d'autres paires de langues. En définitive, les différentes analyses de cette étude se fondent sur l'évaluation quantitative du comportement de plus de 43000 éléments d'origine LD. Une telle quantité de données ne saurait avoir été une mince affaire à gérer et à manipuler, mais aura permis de garantir ultimement l'adéquation des analyses présentées dans cette étude visant à valider ou infirmer les nombreuses propositions théoriques souvent mutuellement contradictoires sur les emprunts en contexte bilingue. Si le lecteur a l'impression que j'insiste beaucoup sur les données - et Poplack le fait aussi dans son ouvrage -, c'est que jusqu'ici les données empiriques ont manqué à montrer le comportement des emprunts et des alternances codiques dans une communauté linguistique donnée.

Toutes les mesures dans cet ouvrage sont finement exécutées, et s'appuient sur la perspective variationniste appliquée à la situation de bilinguisme exposée au chapitre 2, qui conduit notamment à la distinction opérationnelle entre emprunts attestés (attested loanwords), emprunts à occurrence unique (nonce borrowings) et alternances codiques (code-switches), division tripartite qui sous-tend les analyses qui sont présentées dans cet ouvrage.

L'un des points importants dans cette étude est la distinction formelle entre code-switching et borrowing, leurs manifestations et leurs fréquences d'usage chez les locuteurs de la communauté. Le code-switching implique la juxtaposition de séquences d'une langue dans les séquences d'une autre langue, comme dans l'exemple : "Quand il marchait là, il marchait over dead bodies ${ }^{1}$. Bien que cela paraisse ici évident, les travaux sur le code-switching demeurent remplis de controverses. Les travaux sur le terrain du comportement des bilingues suggèrent que ces controverses proviennent de l'incapacité des chercheurs à reconnaitre les différentes manifestations du borrowing. Poplack montre que les locuteurs, d'autre part, font une distinction fondamentale entre les différents types de borrowing et le code-switching. Ainsi, dans l'exemple suivant, "Je groovais comme si j'étais à un show de rap », il est clair que le terme emprunté groove est parfaitement intégré dans la morphologie de la LR. Comment cela peut-il passer inaperçu ? se demande Poplack. Les analyses de Poplack et son équipe exposées dans cet ouvrage, à partir de 13 paires de langues, montrent que les processus linguistiques et sociaux impliqués dans les diverses manifestations du borrowing sont au moins aussi complexes et étonnants que ceux qui sous-tendent le code-switching. Il s'avère que le borrowing est la manifestation principale du mélange linguistique. Pour ce qui est du corpus du français d'Hull-Ottawa, il est plus fréquent que le code-switching par un facteur de 20. Dans les autres paires de langues étudiées, le code-switching est souvent inexistant. Le codeswitching nécessite une bonne connaissance de la LD, afin de juxtaposer correctement une séquence de la LD dans sa LR, alors que le borrowing ne demande pas à l'usager la connaissance de la LD. Combien de mots empruntons-nous de l'italien sans rien connaitre de

1 Cet exemple et le suivant sont tirés d'une entrevue de l'auteure avec François Grosjean (https://www.psychologytoday.com/us/blog/life-bilingual/201711/when-bilinguals-borrow-one-languageanother, consulté le 5/01/2021). 
cette langue : expresso, capuccino, macaroni, etc. ? Il en va de même de mots empruntés à bien d'autres langues. Dans bien d'autres cas aussi, l'emprunteur n'a même pas conscience qu'il utilise des mots de provenance étrangère, tellement ces emprunts sont intégrés et attestés depuis longtemps dans sa propre langue ; c'est le cas par exemple de mots tels que job, fun, cool, chez les locuteurs francophones d'Hull-Ottawa.

Les deux chapitres les plus intéressants de mon point de vue sont les chapitres 8 et 11 .

Le chapitre 8, How nonce borrowings become loanwords, comme le suggère partiellement son titre, cherche à décrire la trajectoire historique d'emprunts à occurrence unique (nonce borrowings), via une forme intermédiaire d'emprunts isolés récurrents (recurrent lone items), aux emprunts établis de LR (loanwords).

L'hypothèse de diffusion (diffusion assumption) soulève la question de savoir dans quelle mesure des éléments isolés d'origine LD introduits dans LR en tant que formes non attestées passeront dans la catégorie d'éléments isolés récurrents, jusqu'à devenir des emprunts établis. Cette hypothèse suggère par ailleurs que des éléments isolés d'origine LD, introduits dans LR en tant qu'emprunts à occurrence unique, gagneront en fréquence et se disperseront à travers la communauté.

Sous cette hypothèse, les faits ne sont pas très probants étant donné la rareté des données disponibles, même si les trois corpus du français précédemment cités ont été mis à profit. Cependant, la découverte importante est que les éléments d'origine LD traversent rarement le test du temps. Et les emprunts à occurrence unique ne se diffusent que rarement, comme voulait le suggérer cette hypothèse.

Une seconde hypothèse est mise à l'épreuve, the Graduality Assumption. Cette hypothèse considère les voies d'intégration de l'évolution des emprunts à occurrence unique, au moyen de quatre diagnostics : l'inflexion verbale, le marquage du pluriel, la réalisation du déterminant, et l'assignation du genre. La découverte la plus intéressante ici est qu'on n'a trouvé aucune preuve que ces éléments étaient introduits graduellement. Tout au contraire, les éléments d'origine LD assument les structures de la grammaire française dès leur introduction. Ceci indique que lorsque les locuteurs accèdent à un élément isolé d'origine étrangère, dans ce casci l'anglais, ceux-ci prennent la décision immédiate de les traiter comme des emprunts ou des alternances de codes. Peu ont recours à cette dernière option. En d'autres termes, les éléments isolés d'origine LD ont tendance à être empruntés, " and borrowings are already introduced as borrowings » (138), même si la vaste majorité d'entre eux ne deviendront jamais des emprunts authentiques (bona fide loanwords). Dans la discussion qui clôt ce chapitre, Poplack considère la très pertinente question : "Why is the linguistic integration of nonce borrowings abrupt when so much linguistic change is gradual? » (139). La réponse, d'abord pour le moins évasive, conduira aux conclusions qui suivent. Quelle que soit l'explication correcte, l'intégration de façon abrupte d'éléments isolés d'origine LD suggère que les locuteurs décident du traitement à donner à un élément de LD au moment même de son incorporation. Ils peuvent opter pour l'emprunter, auquel cas ils assignent à l'élément les éléments grammaticaux de LR, y compris ses propriétés variationnelles. Ceci est en soit remarquable, nous sommes d'accord. Le fait que l'intégration linguistique apparaisse à l'étape de l'occurrence unique confirme que le processus d'emprunt est totalement indépendant de son intégration sociale, telle que mesurée par la récurrence du mot dans le discours d'autres locuteurs et sa dispersion à travers la communauté. L'autre alternative est de simplement laisser le mot d'origine LD tel quel, ce qui implique de l'incorporer avec ses propriétés grammaticales associés, un processus auquel on réfère au codeswitching. La recherche de Poplack confirme, contrairement aux hypothèses répandues, que cela n'arrive presque jamais avec des mots isolés de LD. Enfin, la façon dont les emprunts à occurrence unique (nonce borrowings) deviennent des emprunts intégrés (loanwords), si jamais cela arrive, se réalise par une sorte de contrat social, et non par un processus de transformation linguistique. Ce qui nous amène tout naturellement au chapitre 11, mon second chapitre préféré. 
Le chapitre 11, The social dynamics of borrowing, discute des facteurs extra-linguistiques multi-variationnels des phénomènes d'emprunts analysés dans cet ouvrage. En ayant recours à des facteurs sociolinguistiques habituels, tels que l'âge, le sexe, le niveau d'éducation, et le statut socio-économique, plus d'autres facteurs moins standards tels que la capacité individuelle bilingue (individual bilingual ability), et l'influence environnementale exercée par le quartier de résidence. Il s'agit de déterminer lesquels parmi ces facteurs sont prédictifs des intérêts comportementaux bilingues : la propension d'un locuteur à emprunter en premier lieu, son taux d'emprunt, et sa prédilection pour un type particulier d'emprunt, en particulier emprunts à occurrence unique versus emprunts établis. Comme ce dernier type n'implique généralement pas le recours à LD (ayant été transmis comme partie du lexique natif), les questions clés sont fondamentalement : qui emprunte? qu'est-ce qui est emprunté? à quelle fréquence? et le rôle de la communauté linguistique bilingue dans la détermination de ces tendances. Retenons que ces facteurs et les mesures qui en sont faites confirment la primauté des normes communautaires dans l'introduction, la diffusion, et le traitement linguistique des matériaux empruntés.

Il faut encore dire un mot sur le rôle de la phonétique comme diagnostic pour l'intégration des emprunts (chapitre 10). Contre toute attente, on y apprendra que plutôt que de travailler en tandem, l'intégration phonétique et l'intégration morpho-syntaxique agissent de façon indépendante avec les mots empruntés, et que la variabilité pour ce qui est de la première est individuelle.

Je ne sais pas si cette étude de Poplack mettra fin aux débats acrimonieux auxquels elle fait plusieurs fois référence dans son ouvrage (une liste de travaux publiés sur le sujet serait trop longue pour être énumérée ici), mais je soupçonne que ses contradicteurs devront investir énormément d'efforts pour invalider les résultats présentés ici.

Qui d'autre que Shana Poplack aurait pu nous donner une analyse aussi minutieuse, aboutie?

À lire, à relire et à méditer !

\section{Bibliographie}

Jaafar, Shurooq Talab, Dipima Buragohain \& Harshita Aini Haroon, 2019, "Differences and Classifications of Borrowed and Loan Words in Linguistics Context: A Critical Review", in Ina Suryani \& Dipima Buragohain (eds.), International Languages and Knowledge - Learning in a Changing World, Perlis, Malaysia UniMAP Press, 95-112.

Poplack, Shana, 1989, "The Care and Handling of a Mega-Corpus", in R. W. Fasold \& D. Schiffrin eds., Language Variation and Change, Amsterdam: John Benjamins, 411-51. 


\section{GLOTTOPOL}

Revue de sociolinguistique en ligne

Comité de rédaction : Michaël Abecassis (University of Oxford), Salih Akin (Université de Rouen Normandie), Sophie Babault (Université de Lille), Aude Bretegnier (Université du Mans), Claude Caitucoli, Véronique Castellotti (Université de Tours), Régine DelamotteLegrand (Université de Rouen Normandie), Alexandre Duchêne (Université de Fribourg), Valentin Feussi (Université d'Angers), Robert Fournier (Carleton University, Ottawa), Stéphanie Galligani (Université Grenoble Alpes), Médéric Gasquet-Cyrus (Université AixMarseille), Emmanuelle Huver (Université de Tours), Normand Labrie (Université de Toronto), Foued Laroussi (Université de Rouen Normandie), Benoit Leblanc (Université du Québec à Trois-Rivières), Mylène Lebon-Eyquem (Université de la Réunion), Fabienne Leconte (Université de Rouen Normandie), Gudrun Ledegen (Université de Rennes), Danièle Moore (Simon Fraser University, Vancouver), Clara Mortamet (Université Jean Monnet, Saint Etienne), Alioune Ndao (Université Cheik Anta Diop, Dakar), Isabelle Pierozak (Université de Tours), Cécile Van den Avenne (Université Sorbonne Nouvelle, Paris 3).

Rédactrice en chef : Clara Mortamet.

Directrice de publication : Fabienne Leconte.

Comité scientifique: Michelle Auzanneau (Université de Paris), Margaret Bento (Université de Paris), Jacqueline Billiez (Université de Grenobles Alpes), Philippe Blanchet (Université de Rennes), Jean-Michel Eloy (Université d'Amiens), Françoise Gadet (Université Paris Nanterre), Monica Heller (Université de Moncton), Caroline Juillard (Université de Paris), Jean-Marie Klinkenberg (Université de Liège), Marinette Matthey (Université Grenoble Alpes), Marie-Louise Moreau (Université de Mons-Hainault), Robert Nicolaï (Université Côte d'Azur), Didier de Robillard (Université de Tours), Valérie Spaëth (Université Sorbonne nouvelle), Claude Truchot (Université de Strasbourg), Daniel Véronique (Université AixMarseille).

\section{Comité de lecture pour ce numéro :}

Nathalie Auger, Michèle Auzanneau, Margaret Bento, Stéphane Bonnery, Josiane Boutet, Lucile Cadet, Danièle Cogis, Claudine Garcia-Debanc, Marc Debono Régine Delamotte, JeanFrançois De Pietro, Marie-Laure Elalouf, Valentin Feussi, Laurent Gajo, Emmanuelle Huver, Christian Lagarde, Mylène Lebon-Eyquem, Fabienne Leconte, Gudrun Ledegen, Nadia Maillard, Maira Mamede, Bruno Maurer, Clara Mortamet, Fanny Rink, Valerie Spaëth, Marielle Rispail, Françoise Ropé, Eguzki Urteaga, Cécile Van den Avenne, Daniel Véronique 